\title{
Tail Behavior of Threshold Models with Innovations in the Domain of Attraction of the Double Exponential Distribution
}

\author{
Aliou Diop, Saliou Diouf \\ Laboratoire d'Etudes et de Recherche en Statistique et Développement, \\ Unité de Formation et de Recherche de Sciences Appliquées et Technologie, \\ Université Gaston Berger, Saint-Louis, Sénégal \\ E-mail:aliou.diop@ugb.edu.sn,saliou_diouf@yahoo.fr \\ Received February 15, 2011; revised March 6, 2011; accepted March 9, 2011
}

\begin{abstract}
We consider a two-regime threshold autoregressive model where the driving noises are sequences of independent and identically distributed random variables with common distribution function $F_{i}, i=1,2$ which belongs to the domain of attraction of double exponential distribution. If in addition, $F_{i} \in S_{r}(\gamma)$ i.e. $\lim _{x \rightarrow \infty} \frac{\bar{F}_{i}(x-y)}{\bar{F}_{i}(x)}=\mathrm{e}^{\gamma y}$, for each $x \in \mathbb{R}$, and $\lim _{x \rightarrow \infty} \frac{\overline{F_{i} * F_{i}(x)}}{\bar{F}_{i}(x)}=: d_{i}<\infty$ where $F^{*} G$ denotes the con-
\end{abstract} volution of the distribution function and $\bar{F}=1-F$, we determine the tail behavior of the process and give the exact values of the coefficient.

Keywords: Tail Behavior, Domain of Attraction, Convolution Tails, Stochastic Recurrence Equation, Threshold Autoregressive Model

\section{Introduction}

Switching regimes is stylized facts encountered in financial data analysis, concerning either financial returns, interest rates or volatilities. The threshold autoregressive (TAR) model was introduced by Tong [1] and has since become quite popular in non-linear time series modeling. The TAR model can be seen as a stochastic difference equation. The tail behavior of a stationary solution of such equation has been widely studied in a variety of context.

A result of Kesten [2] shows that the stationary solution to the stochastic recurrence equation has regularly varying distribution, under quite general conditions on the multiplicative coefficient and the noise term. Davis and Resnick [3] treat the bilinear process with regularly varying innovations. Resnick and Willekens [4] consider a stochastic recurrence equation with regularly varying noise.

In these papers, either the multiplier in the stochastic difference equation is a positive random coefficient or the noise term is an independent and identically distri- buted $\mathbb{R}^{+}$- valued random variable. Furthermore, in general, the coefficient and the noise are assumed to be independent. The latter condition is often not satisfied in applications. Diop and Guégan [5] studied the threshold autoregressive stochastic volatility model where the driving noises are sequences of independent and identically distributed regularly varying random variables.

In our framework, the TAR model is a stochastic difference equation where the multiplicative coefficient and the noise term are dependent. The random coefficient model does not necessarily satisfy the positivity condition on the multiplier and the noise term. In addition, the innovations are assumed to belong to the maximum domain of attraction of the double exponential distribution. To our knowledge the literature is not abundant for this framework.

A distribution function $\mathrm{F}$ is in the domain of attraction of the extreme value distribution $\Lambda(x)=\exp \left\{-\mathrm{e}^{-x}\right\}$, $x \in \mathbb{R}$ if there exists $a_{n}>0, b_{n} \in \mathbb{R}(n \geq 1)$ such that

$$
\lim _{n \rightarrow \infty} F^{n}\left(a_{n} x+b_{n}\right)=\Lambda(x), x \in \mathbb{R} .
$$


A distribution $F$ is in the class $S_{r}(\gamma)$ for $\gamma \geq 0$ if $F(x)<1$ for all $x$ and

$$
\lim _{x \rightarrow \infty} \frac{\bar{F}(x-y)}{\bar{F}(x)}=\mathrm{e}^{\gamma y}, \text { for each } y \in \mathbb{R},
$$

and

$$
\lim _{x \rightarrow \infty} \frac{\overline{F^{*} F}(x)}{\bar{F}(x)}=\lim _{x \rightarrow \infty} \frac{\mathbb{P}\left(X_{1}+X_{2}>x\right)}{\mathbb{P}\left(X_{1}>x\right)}=: d<\infty
$$

where $\bar{F}=1-F$ and $F^{*} G$ denote the convolution of the distribution function $F$ and $G$. See Cline [6] and Embrechts [7] for further details on convolution tails. The constant $\mathrm{d}$ is known to equal $2 \mathbb{E}^{\gamma \mathrm{e}^{\gamma}}$. In the sequel, we set $m_{F}(\gamma)=\mathbb{E}^{\gamma X}$ when $F$ is the distribution function of the random variable $X$.

The aim of this paper is to study the tail behavior of a two-regime threshold autoregressive model when the driving noise of each regime has distribution function $F_{i} \in D(\Lambda) \cap S_{r}(\gamma), i=1,2$.

Let us give the motivation to justify why these results are needed to be extended to the proposed class of residual processes. First, the distribution in $S_{r}(\gamma)$ are among others used to model claim size in risk theory Klüppelberg [8], Beard et al.[9], Hogg and Klugman [10], Goldie and Resnick [11]. Second, $D(\Lambda) \cap S_{r}(\gamma)$ is a large class of distributions which constitute the domain of attraction of mean residual lifetimes to the exponential limit law whose behavior is preserved under convolutions. In this paper, precisely we determine the exact value of the coefficient in the tail behavior of the stationary solution when the model is stationary in some regimes and mildly explosive in others.

The rest of this paper is organized as follows: Section 2 describes the model and conditions for strict stationarity are provided. Some preliminary results with respect to the innovation processes are given. Section 3 presents the main results.

\section{The Model}

The threshold autoregressive (TAR) model is defined by the following relation

$$
\alpha_{t}=\left\{\begin{array}{l}
\Phi_{1} \alpha_{t-1}+Z_{t}^{(1)}, \text { if } \quad Y_{t-1} \leq \tau \\
\Phi_{2} \alpha_{t-1}+Z_{t}^{(2)}, \text { if } \quad Y_{t-1}>\tau
\end{array}\right.
$$

where $\tau, \Phi_{i}$ are non random constants and with threshold variable $Y_{t-1}$.

\subsection{Assumptions}

We will use the following assumptions.
$\boldsymbol{H}_{\mathbf{1}^{-}}\left(Z_{1}^{(i)}\right)$ is sequence of independent and identically distributed random variables $(i=1,2)$ and satisfied the following conditions:

$$
\mathbb{E}\left[\log ^{+} Z_{1}^{(i)}\right]<+\infty,
$$

where $\log ^{+} x=\max (0, \log x)$.

$\boldsymbol{H}_{2^{-}}$For each $i=1,2$, the two sequences of random variables $\left(Z_{t}^{(i)}\right)_{t}$ and $\left(Y_{t}\right)_{t}$ are independent and $\left(Z_{t}^{(1)}\right)_{t}$ and $\left(Z_{t}^{(2)}\right)_{t}$ are independent.

$\boldsymbol{H}_{3^{-}}$The sequence of independent and identically distributed random variables $\left(Z_{t}^{(i)}\right)_{t}$ whose common distribution $F_{i}$ is both in the domain of attraction of $\Lambda(x)=\exp \left\{-\mathrm{e}^{-x}\right\}, x \in \mathbb{R}$ and in $S_{r}(\gamma), \gamma \geq 0$ and satisfies the tail balancing condition.

$$
\lim _{x \rightarrow \infty} \frac{\mathbb{P}\left(Z_{1}^{(i)}>x\right)}{\mathbb{P}\left(\left|Z_{1}^{(i)}\right|>x\right)}=p, \quad \lim _{x \rightarrow \infty} \frac{\mathbb{P}\left(Z_{1}^{(i)}<-x\right)}{\mathbb{P}\left(\left|Z_{1}^{(i)}\right|>x\right)}=1-p,
$$

We define $=\mathbb{P}\left(Y_{t} \leq \tau\right), \quad I_{1 t}=1_{\left\{Y_{t-1} \leq \tau\right\}}, \quad I_{2 t}=1-I_{1 t}$. Then Equation (4) can be rewritten as:

where

$$
\alpha_{t}=\Phi_{(t)} \alpha_{t-1}+Z_{t}
$$

$$
\Phi_{(t)}=\Phi_{1} I_{1 t}+\Phi_{2} I_{2 t t} \text { and } Z_{t}=Z_{t}^{(1)} I_{1 t}+Z_{t}^{(2)} I_{2 t} .
$$

We easily check that the tail balancing condition (6) holds for random variables $\left(Z_{k}\right)$ whose distribution function $F=q F_{1}+(1-q) F_{2}$.

\subsection{Preliminary Result}

The Equation (7) is a stochastic difference equation where the pair $\left(\Phi_{(t)}, Z_{t}\right)_{t}$ are sequences of iid $\mathbb{R}^{2}-$ values random variables under $H_{1}$ and $H_{2}$. The next proposition gives the strict stationarity of the process $\left(\alpha_{t}\right)$, defined in (7). The result follows from Theorem 1 of Brandt [12].

Proposition 1 (strict stationarity) Assume $H_{1}$ and $H_{2}$ and suppose that $\left|\Phi_{1}\right|^{q}\left|\Phi_{2}\right|^{1-q}<1$. Then, for all $t \in \mathbb{Z}$, the series $\left(\alpha_{t}\right)_{t}$ defined in (7) admits the following expansion

$$
\alpha_{t}=\sum_{j=0}^{\infty}\left(\prod_{k=0}^{j-1} \Phi_{(t-k)}\right) Z_{t-j},
$$

which converges almost surely. Then the process $\left(\alpha_{t}\right)_{t}$ is the unique strictly stationary solution of (7).

Proposition 2 Let $F=q F_{1}+(1-q) F_{2}$.

1) If $F_{i} \in D(\Lambda), i=1,2$, then $F \in D(\Lambda)$.

2) If $F_{i} \in S_{r}(\gamma), i=1,2, \gamma \geq 0$, then $F \in S_{r}(\gamma)$.

Proof 
Without loss of generality, we can assume that $\frac{\bar{F}_{2}(x)}{\bar{F}_{1}(x)}$, tends to some constant $c \geq 0$ as $x \rightarrow \infty$ which we denote by $\bar{F}_{2} \sim c \bar{F}_{2}$ with $c \geq 0$ Then the proof of 1 ) follows from Proposition 3.3.28 of Embrechts and et al. [13]. Indeed

$$
\frac{\bar{F}(x)}{\bar{F}_{1}(x)}=q+(1-q) \frac{\bar{F}_{2}(x)}{\bar{F}_{1}(x)},
$$

which tends to some positive constant $k>0$ as $x \rightarrow \infty$ Hence $\mathrm{F}$ belongs to $D(\Lambda)$ Now we prove 2).

First, using $\bar{F}_{2} \sim c \bar{F}_{1}$ with $c \geq 0$, it is easy to show by simple calculations that

$$
\frac{\bar{F}(x-y)}{\bar{F}(x)} \rightarrow \mathrm{e}^{\gamma y}, \text { as } x \rightarrow \infty .
$$

It suffices now to show that

$$
\lim _{x \rightarrow \infty} \frac{\overline{F^{*} F}(x)}{\bar{F}(x)}=d<\infty .
$$

Now, we use the decomposition

$$
\begin{aligned}
& \mathbb{P}\left(Z_{1}+Z_{2}>x\right) \\
= & q^{2} \mathbb{P}\left(Z_{1}^{(1)}+Z_{2}^{(1)}>x\right)+q(1-q) \mathbb{P}\left(Z_{1}^{(1)}+Z_{2}^{(2)}>x\right) \\
& +q(1-q) \mathbb{P}\left(Z_{1}^{(2)}+Z_{2}^{(1)}>x\right)+(1-q)^{2} \mathbb{P}\left(Z_{1}^{(2)}+Z_{2}^{(2)}>x\right)
\end{aligned}
$$

Then

$$
\begin{aligned}
& \frac{\mathbb{P}\left(Z_{1}+Z_{2}>x\right)}{\mathbb{P}\left(Z_{1}>x\right)} \\
& =\frac{q^{2} \mathbb{P}\left(Z_{1}^{(1)}+Z_{2}^{(1)}>x\right)}{\mathbb{P}\left(I_{11} Z_{1}^{(1)}+I_{21} Z_{2}^{(1)}>x\right)}+\frac{q(1-q) \mathbb{P}\left(Z_{1}^{(1)}+Z_{2}^{(2)}>x\right)}{\mathbb{P}\left(I_{11} Z_{1}^{(1)}+I_{21} Z_{1}^{(2)}>x\right)} \\
& \quad+\frac{q(1-q) \mathbb{P}\left(Z_{1}^{(2)}+Z_{2}^{(1)}>x\right)}{\mathbb{P}\left(I_{11} Z_{1}^{(1)}+I_{21} Z_{1}^{(2)}>x\right)}+\frac{(1-q)^{2} \mathbb{P}\left(Z_{1}^{(2)}+Z_{2}^{(2)}>x\right)}{\mathbb{P}\left(I_{11} Z_{1}^{(1)}+I_{22} Z_{2}^{(1)}>x\right)}
\end{aligned}
$$

We can write

$$
\begin{aligned}
\frac{\overline{F^{*} F}(x)}{\bar{F}(x)}= & \frac{q^{2} \overline{F_{1}^{*} F_{1}}(x)}{\bar{F}(x)}+\frac{2 q(1-q) \overline{F_{1}^{*} F_{2}}(x)}{\bar{F}(x)} \\
& +\frac{(1-q)^{2} \overline{F_{2} * F_{2}}(x)}{\bar{F}(x)}
\end{aligned}
$$

where $\bar{F}(x)=q \bar{F}_{1}(x)+(1-q) \bar{F}_{2}(x)$.

Since $F_{1}$ and $F_{2}$ belongs to the class $S_{r}(\gamma)$ we have

$$
\lim _{x \rightarrow \infty} \frac{\overline{F_{1}^{*} F_{1}}(x)}{\bar{F}_{1}(x)}=d_{1}<\infty
$$

and

$$
\lim _{x \rightarrow \infty} \frac{\overline{F_{2}^{*} F_{2}}(x)}{\bar{F}_{2}(x)}=d_{2}<\infty .
$$

Then

$$
q^{2} \frac{\overline{F_{1}^{*} F_{1}}(x)}{\bar{F}(x)} \rightarrow \frac{q^{2} d_{1}}{q+c(1-q)} .
$$

Similarly, we have

$$
\frac{(1-q)^{2} \overline{F_{2} * F_{2}}(x)}{\bar{F}(x)} \rightarrow \frac{(1-q)^{2} c d_{2}}{q+c(1-q)}
$$

Using Theorem 1 of Cline [6], we show that

$$
\frac{q(1-q) \overline{F_{1}^{*} F_{2}}(x)}{\bar{F}(x)} \rightarrow \frac{q(1-q)\left(d_{1}+c d_{2}\right)}{q+c(1-q)} .
$$

The proposition is entirely demonstrated.

\section{Main Results}

Our aim in this section is to establish the tail behavior of the stationary distribution of $\left(\alpha_{t}\right)_{t}$ defined in (4).

Theorem 1 Let $\left(\alpha_{t}\right)_{t}$ be the stationary solution of Equation (7) and the process $\left(Z_{t}\right)_{t}$ be an iid sequence of random variables with common distribution $F_{i} \in D(\Lambda) \cap S_{r}(\gamma)$ satisfying (6). Suppose that the assumptions of Proposition 1 hold. Then the tail behavior of the stationary distribution of $\left(\alpha_{t}\right)_{t}$ is

$$
\begin{aligned}
& \lim _{x \rightarrow \infty} \frac{\mathbb{P}\left(\alpha_{t}>x\right)}{\bar{F}(x)} \\
= & \left(\sum_{i=0}^{n} \beta_{i} m_{G_{0} *_{G_{1}} * \cdots * G_{i-1}(\gamma)} m_{G_{i+1}(\gamma)} \cdots m_{G_{n}(\gamma)}\right) \\
& \times\left(\sum_{i=0}^{n+1} m_{\alpha_{t}}\left(\gamma \Phi_{1}^{i} \Phi_{2}^{n+1-i}\right) p_{n+1, i}\right),
\end{aligned}
$$

where

$$
m_{G_{j}}(\gamma)=\left(\sum_{k=0}^{j} m_{F}\left(\gamma \Phi_{1}^{k} \Phi_{2}^{j-k}\right)\right) p_{j, k}, p_{j, k}=C_{j}^{k} q^{k}(1-q)^{j-k}
$$

$m_{G_{0}{ }^{*} G_{1} * \ldots * G_{i}}$ can be computed using the following recursion formula:

$$
\left\{\begin{array}{l}
m_{G_{0}^{i}}(\gamma)=q m_{F_{1}}(\gamma) m_{F}\left(\gamma \Phi_{1}\right)+(1-q) m_{F_{2}}(\gamma) m_{F}\left(\gamma \Phi_{2}\right) \\
m_{G_{0}^{i+1}}(\gamma)=q m_{F_{1}}(\gamma) m_{G_{0}^{i}}(\gamma)+(1-q) m_{F_{2}}(\gamma) m_{G_{0}^{i}}(\gamma)
\end{array}\right.
$$

$$
\mathrm{m}_{\mathrm{G}_{0}^{\mathrm{i}}}(\gamma)=m_{G_{0} * G_{1} * \ldots * G_{i}}(\gamma)
$$

and 


$$
\beta_{k}=\left\{\begin{array}{lr}
q^{k}, & \text { if } \Phi_{1}=1,\left|\Phi_{2}\right|<1, \\
p^{-1} q^{k}\left(p \delta_{k}+(1-p) \delta_{k+1}\right), & \text { if } \Phi_{1}=-1,\left|\Phi_{2}\right|<1, \\
(1-q)^{k}, & \text { if } \Phi_{2}=1,\left|\Phi_{1}\right|<1, \\
p^{-1}(1-q)^{k}\left(p \delta_{k}+(1-p) \delta_{k+1}\right), & \text { if } \Phi_{2}=-1,\left|\Phi_{1}\right|<1, \\
0, & \text { if }\left|\Phi_{1}\right|<1,\left|\Phi_{2}\right|<1,
\end{array}\right.
$$

with

$$
\delta_{k}= \begin{cases}1, & \text { if } k \text { even } \\ 0, & \text { if } k \text { old }\end{cases}
$$

Remark: We may give an financial example of model (4) introduced by Breidt [14] for a financial return $Y_{t}$ defined by the following relation

$$
Y_{t}=\sigma \exp \left(\frac{\alpha_{t}}{2}\right) \varepsilon_{t}
$$

Where $\alpha_{t}$ is an open-loop threshold autoregressive process (Tong [15], p. 101)

$$
\alpha_{t}= \begin{cases}\Phi_{1} \alpha_{t-1}+Z_{t}^{(1)}, & \text { if } Y_{t-1} \leq 0 \\ \Phi_{2} \alpha_{t-1}+Z_{t}^{(2)}, & \text { if } Y_{t-1}>0\end{cases}
$$

The model is called a threshold autoregressive stochastic volatility model (TARSV). The log-volatility process $\left(\alpha_{t}\right)_{t}$ has a piecewise linear structure. It switches between two first-order autoregressive process according to the sign of the previous return. In this framework, $\sigma$ is positive constant and $\left(\varepsilon_{t}\right)_{t}$ is a sequence of independent and identically distributed random variables with zero mean and its variance is taken to be one. When either $\left|\Phi_{1}\right|=1$ and $\left|\Phi_{2}\right| \neq 1$ or $\left|\Phi_{2}\right|=1$ and $\left|\Phi_{1}\right| \neq 1$, the process defined in (15) is stationary in some regimes and mildly explosive in others. These models are stationary in some regimes and mildly explosive in others. See Gongalo and Montesinos [16]. Gouriéroux and Robert [17] studied the ACR(1) process where there is a switching between white noise and a random walk.

Before proving Theorem1 we establish three lemmas. The next lemma is due to Davis and Resnick [18]. Its proof will then be omitted. The second lemma is an extension of Proposition 1.2 in Davis and Resnick [18] where the hypothesis of independence is relaxed. See also Cline [6]. They are needed for the proof of the tail behavior of $\left(\alpha_{t}\right)$.

\section{Lemma 1}

Suppose $F \in D(\Lambda)$ so the Balkema and de Haan[19] representation holds

$$
\bar{F}(x)=\theta(x) \exp \left\{-\int_{Z_{0}}^{x} \frac{1}{f(t)} \mathrm{d} t\right\},
$$

for some $z_{0}$ and $x>z_{0}$ where $\theta(x) \rightarrow \theta \in(0, \infty)$ as $x \rightarrow \infty, f>0$ is absolutely continous on $\left(z_{0}, \infty\right)$ with density $f^{\prime}$ and $\lim f^{\prime}(u)=0$. Given $\varepsilon>0$, there exists $x_{0}=x_{0}(\varepsilon)$ such that for $x \geq x_{0}$

$$
\frac{\bar{F}\left(c^{-1} x\right)}{\bar{F}(x)} \leq(1+\varepsilon)\left(\frac{f(x)}{x}\right)^{\frac{1}{\varepsilon}}\left(\frac{c}{\varepsilon(1-c)}\right)^{\frac{1}{\varepsilon}},
$$

For any $0<c<1$.

This following lemma is quite general since it does not require the hypothesis of independent between the $Y_{i}$ 's.

\section{Lemma 2}

Let $\left\{Y_{i}, 1 \leq i \leq n\right\}$ be random variables with distribution function $G_{i}$ and suppose $F \in S_{r}(\gamma)$. If

$$
\lim _{x \rightarrow \infty} \frac{\mathbb{P}\left(Y_{i}>x\right)}{\bar{F}(x)}=\alpha_{i} \in[0, \infty]
$$

for $i=1, \cdots, n$ then for all $n \geq 2$,

$$
\begin{aligned}
& \lim _{x \rightarrow \infty} \frac{\mathbb{P}\left(\sum_{i=1}^{n} Y_{i}>x\right)}{\bar{F}(x)} \\
& =\sum_{i=1}^{n} \alpha_{i} m_{G_{1} * G_{2} * \cdots * G_{i-1}}(\gamma) m_{G_{i+1}}(\gamma) \cdots m_{G_{n}}(\gamma)
\end{aligned}
$$

\section{Proof}

When $n=2$, then this lemma is the formulation of Theorem 1 of Cline [6]. When $n>2$, set $S n=\sum_{i=1}^{n} Y_{i}$ and $G$ its distribution function. Applying (19), when $n=2$, we have

$$
\begin{aligned}
& \lim _{x \rightarrow \infty} \frac{\mathbb{P}\left(\sum_{i=1}^{n} Y_{i}>x\right)}{\bar{F}(x)}=\lim _{x \rightarrow \infty} \frac{\mathbb{P}\left(S_{n}+Y_{n+1}\right)}{\bar{F}(x)} \\
& =\alpha_{n+1} m_{G}(\gamma)+\left(\sum_{i=1}^{n} \alpha_{i} m_{G_{i}^{i-1}}(\gamma) m_{G_{i+1}}(\gamma) \cdots m_{G_{n}}(\gamma)\right) m_{G n+1}(\gamma) \\
& =\sum_{i=1}^{n+1} \alpha_{i} m_{G_{1}^{i-1}}(\gamma) m_{G_{i+1}}(\gamma) \cdots m_{G_{n+1}}(\gamma) .
\end{aligned}
$$

with

$$
m_{G_{0}^{i}}(\gamma)=m_{G_{0} * G_{1} * \ldots * G_{i}}(\gamma)
$$

\section{Lemma 3}

$$
\lim _{x \rightarrow \infty} \frac{\mathbb{P}\left(\sum_{j=n+1}^{\infty}\left(\prod_{k=0}^{j-1} \Phi_{(t-k)}\right) Z_{t-j}>x\right)}{\mathbb{P}\left(Z_{t} \geq x\right)}=0 .
$$

\section{Proof}

For $\varepsilon>0$ and for all $\mathrm{b}$ such that

$$
1<b^{1 / \varepsilon}<\frac{1}{\mathbb{E}\left|\Phi_{(1)}\right|^{1 / \varepsilon}},
$$


We have

$$
\begin{aligned}
& \mathbb{P}\left(\sum_{j=n+1}^{\infty}\left(\prod_{k=0}^{j-1} \Phi_{(t-k)}\right) Z_{t-j}>x\right) \\
& \leq \sum_{j=n+1}^{\infty} \sum_{k=0}^{j} \mathbb{P}\left\{\left|\Phi_{1}^{k} \Phi_{2}^{j-k}\right|\left|Z_{t-j}\right| b^{n-j}(b-1) x\right\} p_{j, k},
\end{aligned}
$$

where

$$
p_{j, k}=C_{j}^{k} q^{k}(1-q)^{j-k} .
$$

Using the tail balancing condition (6), we have

$$
\begin{aligned}
& \sum_{k=0}^{j} \frac{\mathbb{P}\left(\left|Z_{t-j}\right|>x \delta_{k}\right)}{\mathbb{P}\left(Z_{t-j}>x \delta_{k}\right)} \frac{\mathbb{P}\left(Z_{t-j}>x \delta_{k}\right)}{\mathbb{P}\left(Z_{t}>x\right)} p_{j, k} \\
& =\sum_{k=0}^{j} \frac{\bar{F}\left(x \delta_{k}\right)}{\bar{F}(x)} p^{-1} p_{j, k},
\end{aligned}
$$

where $\delta_{k}=b^{n-j}(b-1)\left|\Phi_{1}^{k} \Phi_{2}^{j-k}\right|^{-1}$. Since $\mathrm{b}>1$, it is readly seen that $\mathrm{b}>1$, for all. $j>n$ and $>k$. Hence applying Lemma 1 with $c=\delta_{k}^{-1}$, given $\varepsilon>0$, there exists $x_{0}$ such that for $x>x_{0}$, we have

$$
\sum_{k=0}^{j} \frac{\bar{F}\left(x \delta_{k}\right)}{\bar{F}(x)} p_{j, k} \leq \sum_{k=0}^{j}(1+\varepsilon)\left(\frac{f(x)}{x}\right)^{\frac{1}{\varepsilon}}\left(\frac{\delta_{k}^{-1}}{\varepsilon\left(1-\delta_{k}^{-1}\right)}\right)^{\frac{1}{\varepsilon}} p_{j, k} .
$$

Now following the proof of Proposition1.1 of Davis and Resnick [18], we give an upper bound of

$$
K\left(\frac{f(x)}{x}\right)^{\frac{1}{\varepsilon}} \sum_{j=n+1 k=0}^{\infty} \sum_{k}^{j}\left[\left(b^{n-j}(b-1)\right)^{-1}\left(\left|\Phi_{1}^{k} \Phi_{2}^{j-k}\right|\right)\right]^{\frac{1}{\varepsilon}} p_{j, k}
$$

Observe that

$$
\begin{aligned}
& \sum_{k=0}^{j}\left[\left(b^{n-j}(b-1)\right)^{-1}\left(\left|\Phi_{1}^{k} \Phi_{2}^{j-k}\right|\right)\right]^{\frac{1}{\varepsilon}} p_{j, k} \\
& =\left(b^{n}(b-1)\right)^{-\frac{1}{\varepsilon}}\left(\mathbb{E}\left|b \Phi_{(1)}\right|^{\frac{1}{\varepsilon}}\right)^{j}
\end{aligned}
$$

This implies

$$
\begin{aligned}
& \mathbb{P}\left(\sum_{j=n+1}^{\infty}\left(\prod_{k=0}^{j-1} \Phi_{(t-k)}\right) Z_{t-j}>x\right) \\
& \leq \sum_{j=n+1 k=0}^{\infty} \sum_{k}^{j} \mathbb{P}\left\{\left|\Phi_{1}^{k} \Phi_{2}^{j-k}\right|\left|Z_{t-j}\right|>b^{n-j}(b-1) x\right\} \\
& \leq K\left(\frac{f(x)}{x}\right)^{\frac{1}{\varepsilon}} \sum_{j=n+1}^{\infty}\left(\mathbb{E}\left|b \Phi_{(1)}\right|^{\frac{1}{\varepsilon}}\right)^{j} .
\end{aligned}
$$

Since

$$
\mathbb{E}\left|b \Phi_{(1)}\right|^{\frac{1}{\varepsilon}}<1 \text { and } \lim _{x \rightarrow \infty} \frac{f(x)}{x}=\lim _{x \rightarrow \infty} f^{\prime}(x)=0
$$

The result follows.

\section{Proof of Theorem 1}

Set

$$
\begin{gathered}
S_{n}=\sum_{j=0}^{n}\left(\prod_{k=0}^{j-1} \Phi_{(t-k)}\right) Z_{t-j}, \\
R_{n}=\sum_{j=n+1}^{\infty}\left(\prod_{k=0}^{j-1} \Phi_{(t-k)}\right) Z_{t-j}, \\
Y_{n}=\left(\prod_{k=0}^{j-1} \Phi_{(t-k)}\right) Z_{t-j} .
\end{gathered}
$$

Denote by $G_{j}$ the distribution function of $Y_{j}$. First, it is easy to check that the moment generating function $Y_{j}$ exists and is given by

$$
m_{G_{j}}(\gamma)=\sum_{i=0}^{j} m_{F}\left(\gamma \Phi_{1}^{i} \Phi_{2}^{j-i}\right) p_{j, i}
$$

Next note that $R_{n}=\Phi_{(t)} \Phi_{(t-1)} \cdots \Phi_{(t-n)} \alpha_{(t-n-1)}$. Using the independence between $\left\{\Phi_{(t)}, \Phi_{(t-1)}, \cdots, \Phi_{(t-n)}\right\}$ and $\alpha_{(t-n-1)}$, we can compute the moment generating function of $R_{n}$ which we denote by $m_{R_{n}}$,

$$
m_{R_{n}}(\gamma)=\sum_{i=0}^{n+1} m_{\alpha_{(t-n-1)}}\left(\gamma \Phi_{1}^{i} \Phi_{2}^{n+1-i}\right) p_{n+1, i}
$$

where $m_{\alpha_{t}}$ is the moment generating function of $\alpha_{t}$. By the stationarity of $\left(\alpha_{t}\right)$, we get

$$
m_{R_{n}}(\gamma)=\sum_{i=0}^{n+1} m_{\alpha_{t}}\left(\gamma \Phi_{1}^{i} \Phi_{2}^{n+1-i}\right) p_{n+1, i}
$$

Using the rapidly variation of $\bar{F}$ and the tail balancing condition (6), we can establish that

$$
\begin{aligned}
& \lim _{x \rightarrow \infty} \frac{\mathbb{P}\left(Y_{i}>x\right)}{\bar{F}(x)} \\
& =\lim _{x \rightarrow \infty} \sum_{k=0}^{j} \frac{\mathbb{P}\left(\Phi_{1}^{k} \Phi_{2}^{j-k} Z_{t-j}>x\right)}{\bar{F}(x)} p_{j, k} \\
& =\beta_{j}
\end{aligned}
$$

$\beta_{j}$ is given by (12).

By Lemma 2, we have

$$
\begin{aligned}
& \lim _{x \rightarrow \infty} \frac{\mathbb{P}\left(S_{n}>x\right)}{\bar{F}(x)} \\
& =\sum_{i=1}^{n} \alpha_{i} m_{G_{1} * G_{2} * \cdots * G_{i-1}}(\gamma) m_{G_{i+1}}(\gamma) \cdots m_{G_{n}}(\gamma)
\end{aligned}
$$

Moreover, by lemma 3 


$$
\lim _{x \rightarrow \infty} \frac{\mathbb{P}\left(R_{n}>x\right)}{\bar{F}(x)}=0
$$

Combining (23) and (24) and applying again Lemma 2, we obtain the desired result.

\section{Acknowledgements}

These results were obtained thanks to the support of AIRES-Sud, a programme from the French Ministry of Foreign and European Affairs implemented by the Institut de Recherche pour "le Développement (IRD-DSF)". The authors acknowledge grants from "Ministère de la Recherche Scientifique" of Senegal.

\section{References}

[1] H. Tong, "Non-Linear Time Series: A Dynamical System Approach," Oxford University Press, New York, 1990.

[2] H. Kesten, "Random Difference Equations and Renewal Theory for Products of Random Matrices," Acta Mathematica, Vol. 131, No. 4, 1973, pp. 207-248. doi:10.1007/BF02392040

[3] R. A. Davis and S. I. Resnick, "Limit Theory for Bilinear Process with Heavy-Tailed," The Annals of Applied Probability, Vol. 6, No. 4, 1996, pp. 1191-1210. doi:10.1214/aoap/1035463328

[4] S. I. Resnick and E. Willekens, "Moving Averages with Random Coefficient Autoregressive Models," Stochastic Models, Vol. 7, No. 4, 1991, pp. 511-525.

[5] A. Diop and D. Guégan, "Tail Behavior of Threshold Autoregressive Stochastic Volatility Model," Extremes, Vol. 7,No. 4, 2004, pp. 367-375. doi:10.1007/s10687-004-3482-y

[6] D. B. H. Cline, "Convolution Tails, Products Tails and Domains of Attraction," Probability Theory and Related Fields, Vol. 72, No. 4, 1986, pp. 529-557. doi:10.1007/BF00344720

[7] P. Embrechts, "Subexponential Distribution Functions and Their Applications: A Review," Proceedings of the Seventh Conference Probability Theory, Brasov, 29 August-4 September 1982, pp. 125-136.
[8] C. Klüppelberg, "Subexponential Distributions and Integrated Tails," Journal of Applied Probability, Vol. 25, No. 1, 1988, pp. 132-141. doi:10.2307/3214240

[9] R. E. Beard, T. Pentikainen and E. Pesonen, "Risk Theory," Chapman \& Hall, London, 1984.

[10] R. V. Hogg and S. A. Klugman, "Loss Distributions," Wiley, New York, 1984. doi:10.1002/9780470316634

[11] C. M. Goldie and S. I. Resnick, "Distributions That Are Both Subexponential and in the Domain of Attraction of an Extreme-Value Distribution," Advances in Applied Probability, Vol. 20, No. 4, 1988, pp. 706-718. doi: $10.2307 / 1427356$

[12] A. Brandt, "The Stochastic Equation $Y_{n+1}=A_{n} Y_{n}+B_{n}$ with Stationary Coefficients," Advances in Applied Probability, Vol. 18, No. 1, 1986, pp. 211-220. doi: $10.2307 / 1427243$

[13] P. Embrechts, C. Kluppelberg and T. Mikosch, "Modelling Extremal Events for Insurance and Finance," Springer Verlag, Berlin, 1997.

[14] F. J. Breidt, "A Threshold Autoregressive Stochastic Volatility Model," VI Latin American Congress of Probability and Mathematical Statistics(CLAPEM), Valparaiso, 1996.

[15] H. Tong, "On a Threshold Model, Pattern Recognition and Signal Processing," C. H. Chen, Ed., Sijhoff \& Noordoff, Amsterdam, 1990.

[16] J. Gonzalo and R. Montesinos, "Threshold Stochastic Unit Root Models," Manuscript, Universidad Carlos III, 2002.

[17] C. Gouriéroux and C. Y. Robert, "Stochastic Unit Root Models," Econometric Theory, Vol. 22, No. 6, 2006, pp. 1052-1090. doi: 10.1017/S0266466606060518

[18] R. Davis and S. Resnick "Extremes of Moving Averages of Random Variables from the Domain of Attraction of the Double Exponential Distribution," Stochastic Processes and their Applications, Vol. 30, No. 1, 1988, pp. 41-68. doi:10.1016/0304-4149(88)90075-0

[19] A. A. Balkema and L. De Haan, "On R. Von Mises's Condition for the Domain of Attraction of $\operatorname{Exp}\left(-\mathrm{e}^{-\mathrm{x}}\right)$," The Annals of Mathematical Statistics, Vol. 43, No. 4, 1972, pp. 1352-1354. doi:10.1214/aoms/1177692489 